\title{
Effects of Respiratory Liver Motion on Heating for Gated and Model-Based Motion-Compensated High-Intensity Focused Ultrasound Ablation
}

\author{
Erik-Jan Rijkhorst ${ }^{1}$, Ian Rivens ${ }^{2}$, Gail ter Haar $^{2}$, \\ David Hawkes ${ }^{1}$, and Dean Barratt ${ }^{1}$ \\ 1 Centre for Medical Image Computing, University College London, London, UK \\ 2 The Institute of Cancer Research, Sutton, Surrey, UK
}

\begin{abstract}
Purpose: To quantify the effects of respiratory motion on high-intensity focused ultrasound heating of liver tissue by comparing the simulated ablation using a conventional respiratory gating versus a MR-model-based motion compensation approach.

Methods: To measure liver motion, dynamic free-breathing abdominal MR scans were acquired for five volunteers. Deformable registration was used to calculate continuous motion models, and tissue heating at a moving single focus was computed in $3-\mathrm{D}$ by solving the bioheat equation. Ablated volume ratios with respect to the static case, $\mathcal{V}^{\text {ab }}$, were determined for a range of exposure times $t^{\exp }$ and heating rates $r$.

Results: To achieve $\mathcal{V}^{\mathrm{ab}}>90 \%$ required $t^{\exp }<0.5 \mathrm{~s}$ and $r>120^{\circ} \mathrm{C} / \mathrm{s}$ when gating, whereas $t^{\exp }<1 \mathrm{~s}$ and $r>60^{\circ} \mathrm{C} / \mathrm{s}$ for motion-compensation.

Conclusions: Accurate compensation for respiratory motion is important for efficient tissue ablation. Model-based motion compensation allows substantially lower heating rates than gating, reducing the risk of skin burns and focal boiling.
\end{abstract}

Keywords: motion compensation, HIFU, heating, liver.

\section{Introduction}

High-intensity focused ultrasound (HIFU) ablation is being increasingly studied as a non-invasive treatment option for liver cancer [1-3]. However, liver respiratory motion can be up to a few centimetres under normal free-breathing conditions [4, 5]. Therefore, conventional gating methods lead to very lengthy treatment times, whereas to facilitate continuous ablation, some form of image guidance to steer the HIFU focus needs to be applied.

Recently reported MR-guided approaches show promising results [3], but these are logistically difficult and relatively expensive techniques, requiring specialist equipment. Ultrasound guidance based on direct speckle tracking may have limited application in practice due to the presence of ribs in the beam path [1, 2]. Moreover, the target location still needs to be identified during the procedure, typically using information from a pre-procedural CT or MR scan.

G. Fichtinger, A. Martel, and T. Peters (Eds.): MICCAI 2011, Part I, LNCS 6891, pp. 605-612, 2011. (C) Springer-Verlag Berlin Heidelberg 2011 
The model-based motion compensation approach proposed in this paper overcomes some of these limitations by predicting liver motion using a continuous motion model. In this method, alignment of the patient's rigid anatomy with the model's co-ordinate system results in a known target location. In addition, temporal synchronisation of the motion model with the subject's breathing by means of a surrogate respiratory signal is required. This can be achieved, for instance, using a separate diagnostic ultrasound probe to track diaphragm motion during treatment at an oblique angle from below the ribs.

Although the need for real-time organ motion compensation is widely acknowledged, the impact of residual liver motion for different image-guidance schemes in terms of thermal dose has received little attention to date. The aim of this work was to compare the performance of a conventional gated approach with a model-based motion compensation method. To achieve the same ablated volume, gating requires shorter exposure times and higher heating rates than motion-compensated HIFU, but undesired effects such as skin burns and focal boiling may then become a significant limitation. To compute the required heating rates, continuous motion models using deformable image registration of dynamic MR sequences obtained from five human volunteers were calculated. Both gated and model-guided HIFU treatments in the presence of respiratory liver motion were simulated by solving the bioheat equation, and the thermal dose and corresponding ablated volumes were computed for a range of heating rates and exposure times.

\section{Materials and Methods}

\subsection{Free-Breathing MR Scans}

Free-breathing MR scans with a field-of-view covering the whole abdomen were acquired for five volunteers. A balanced-SSFP sequence (TR/TE=4.3/1.46 ms, $30^{\circ}$ flip angle) was used to obtain high-resolution $4 \mathrm{D}$ dynamic scans during free breathing using a 1.5 T MR scanner (Philips Achieva, Best, The Netherlands) at Guy's Hospital, London. Parallel imaging with a 32-channel coil array using a SENSE acceleration factor of 4.6 resulted in scan times of approximately one second per dynamic volume. Since the largest liver motion normally occurs in the sagittal plane [4, 5], the highest reconstruction resolution of $1.4 \times 1.4 \mathrm{~mm}$ was chosen in this plane, resulting in a slice thickness of $4 \mathrm{~mm}$. For each volunteer, first a set of normal breathing scans were acquired, followed by a set obtained during deeper breathing. Each set contained a total of $N^{\text {acq }}=25$ volumes, covering $\sim 4$ breathing cycles and took $\sim 20$ s to acquire.

\subsection{Deformable Registration of the Dynamic Scans}

Tissue displacements throughout the liver were computed by registering each volume within a set of dynamic MR scans to a reference volume using a non-rigid fluid registration method [6]. This method solves the time-dependent NavierLamé equations for a compressible viscous fluid resulting in a diffeomorphic 

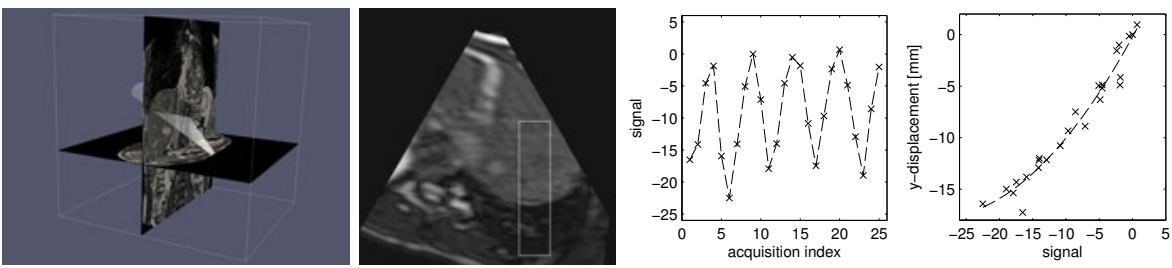

Fig. 1. Geometry of the virtual ultrasound sector used to re-slice the dynamic MR scans (1st panel). Example of a diaphragm navigator window manually located in a slice taken through the reference dynamic MR volume at the interventional ultrasound location (2nd panel) and corresponding surrogate respiratory signal (3rd panel). The 4 th panel shows an example of a motion model fit of y-displacement data as a function of signal value for a single voxel location.

transformation. The registration is driven by image-derived forces and employs a full multi-grid scheme. The volume corresponding to the breathing phase closest to the time of full exhale was chosen as the reference volume. Registering this volume to all other volumes resulted in a set of $N^{\text {acq }}$ displacement fields, denoted by $\boldsymbol{u}_{i}(\boldsymbol{r})$, with $\boldsymbol{r}$ denoting the 3 -D voxel location within the volumes.

To assess the deformable registration accuracy, corresponding anatomical landmarks, such as vessels and vessel bifurcations, were picked manually in each dynamic scan, resulting in a set of $N^{\text {acq }}$ points for each location. Landmark points were identified for each volunteer at five different locations distributed throughout the liver. The slice thickness in the lateral direction was $4 \mathrm{~mm}$, and, because of the poor image quality of the dynamic scans, we did not attempt to quantify motion in the lateral direction. Consequently, only those landmarks clearly visible in a single sagittal plane over the complete dynamic sequence were chosen for the analysis. The target registration error (TRE) was calculated as the root-mean-square distance between the landmark points in the reference volume and all other volumes, following registration.

\subsection{Surrogate Respiratory Signal}

To synchronise a motion model with a subject's breathing during treatment, we assume a diagnostic ultrasound probe is used to acquire dynamic B-mode images of the moving diaphragm at an oblique angle from below the ribs. The motion model is computed using a corresponding diaphragm signal, obtained by re-slicing the dynamic MR volumes at the same location as used during treatment (see Fig. 1). A navigator window was placed over the dome of the right hemi-diaphragm, and successive navigator images were aligned to a reference navigator image at the time of full exhale. This was done using a 1-D translational registration method employing a gradient descent optimisation, and the mean of squared intensity differences as the similarity measure. The resulting 1-D displacements, $s_{i}\left(i=1, \ldots, N^{\text {acq }}\right)$, were used as the surrogate respiratory signal (Fig. 1). 


\subsection{Liver Motion Models}

To express continuous liver motion as a function of the respiratory signal $s$, a smooth function (see Fig. 1, rightmost panel) is fitted to the discrete registration derived displacements $\boldsymbol{u}$, denoted by

$$
\boldsymbol{u}_{i}(\boldsymbol{r}) \equiv \boldsymbol{u}\left(\boldsymbol{r}, s_{i}\right), \text { with } i=1, \ldots, N^{\text {acq }} .
$$

Second order polynomials, given by

$$
\hat{\boldsymbol{u}}(\boldsymbol{r}, s)=\boldsymbol{\alpha}_{1}(\boldsymbol{r})+\boldsymbol{\alpha}_{2}(\boldsymbol{r}) s+\boldsymbol{\alpha}_{3}(\boldsymbol{r}) s^{2},
$$

were used as the motion model fitting function to approximate the data sets. Note that the motion model coefficients $\boldsymbol{\alpha}$ are vector quantities defined at each voxel location $\boldsymbol{r}$. Inserting Eq. (11) into (2) results in an over-determined system of linear equations, given by

$$
\left[\begin{array}{c}
\boldsymbol{u}_{1}(\boldsymbol{r}) \\
\vdots \\
\boldsymbol{u}_{N_{\text {acq }}}(\boldsymbol{r})
\end{array}\right]=\left[\begin{array}{ccc}
1 & s_{1} & s_{1}^{2} \\
\vdots & \vdots & \vdots \\
1 & s_{N^{\mathrm{acq}}} & s_{N^{\mathrm{acq}}}^{2}
\end{array}\right]\left[\begin{array}{c}
\boldsymbol{\alpha}_{1}(\boldsymbol{r}) \\
\boldsymbol{\alpha}_{2}(\boldsymbol{r}) \\
\boldsymbol{\alpha}_{3}(\boldsymbol{r})
\end{array}\right]
$$

From this, the motion model coefficients were determined by computing the Moore-Penrose pseudo-inverse using singular value decomposition.

\subsection{Thermal Model of Tissue Heating}

The evolution of the tissue temperature $T$ as a function of time was computed in 3-D using the implicit Crank-Nicolson finite difference method for solving the bioheat equation [7, 8]

$$
\rho C \frac{\partial T}{\partial t}=K \nabla^{2} T-W_{b} C_{b}\left(T-T_{b}\right)+q(t),
$$

with $\rho$ the mass density, $C$ the specific heat, and $K$ the thermal conductivity of the tissue. Cooling due to perfusion is modelled by the second term on the right hand side, with $C_{b}$ the specific heat, $W_{b}$ the perfusion rate, and $T_{b}$ the temperature of the blood. Table 1 presents the material parameters which were kept constant in the simulations. The HIFU energy density $q(t)$ was computed from the intensity profile obtained using the Rayleigh integral for a spherical

Table 1. Material parameters used in the heating simulations [8, 10]

\begin{tabular}{l|l|l} 
parameter description & value & unit \\
\hline liver specific heat & $C=3770$ & $\mathrm{~J} \mathrm{~kg}^{-1}{ }^{\circ} \mathrm{C}^{-1}$ \\
liver thermal conductivity & $K=0.508$ & $\mathrm{~W} \mathrm{~m}$ \\
blood perfusion rate & $W_{b} \mathrm{C}^{-1}$ \\
blood specific heat & $C_{b}=3770$ & $\mathrm{~kg} \mathrm{~m}^{-3} \mathrm{~s} \mathrm{~kg}^{-1}$ \\
blood temperature & $T_{b}=37$ & ${ }^{\circ} \mathrm{C}$ \\
\hline
\end{tabular}


bowl transducer [8] with a focal length of $15 \mathrm{~cm}$ and a radius of $5 \mathrm{~cm}$. The dependence of the energy density $q$ on time $t$ in Eq. 4 denotes the effects of motion on heating. This was implemented by using the motion model coefficients $\boldsymbol{\alpha}$ from Eq. 3 to compute local displacements with which to translate $q$ relative to the computational domain. To quantify heating effects we use the concept of thermal dose [9], given by

$$
\mathrm{TD}=\sum_{t_{0}}^{t_{\exp }} R^{43-T} \Delta t,
$$

with $R=0.25$ for $T<43^{\circ} \mathrm{C}$, and $R=0.5$ for $T>43^{\circ} \mathrm{C}$, and $\Delta t$ the time step used in the heating simulations. Tissue was assumed to be fully ablated when $\mathrm{TD} \geq 240$ cumulative equivalent minutes.

\subsection{Simulated Treatment Scenarios}

Two scenarios for HIFU treatment during free-breathing were simulated: respiratory gating and continuous MR-model-based motion compensation. For both methods, respiratory liver motion as it occurs during an intervention was simulated using a subject-specific motion model, derived from a set of dynamic MR scans obtained during normal breathing. For the gating approach, ablation was gated symmetrically around the time of full exhale in the breathing cycle.

For the motion compensation method, the HIFU focus was assumed to be steered according to a model-based prediction of local liver motion. Although it would be more realistic to simulate focus steering using a transducer array, for this initial study we opted to simply translate the heating pattern generated by a single bowl transducer. The prediction model was computed from a second set of dynamic MR scans, obtained during deeper breathing a few minutes after the first set was acquired. In practice, the prediction model can be synchronised during treatment to the subject's breathing by means of a navigator signal obtained using real-time ultrasound imaging of the diaphragm (see Fig. 2). In our simulations motion correction was implemented by subtracting the predicted motion from the simulated subject motion, resulting in residual guidance errors representative of those that may occur in practice.

To take into account variations in motion occurring in different parts of the liver, three different simulated ablation locations were chosen. The treatment depth was set to $5 \mathrm{~cm}$ in the anterior-posterior direction, and the location of

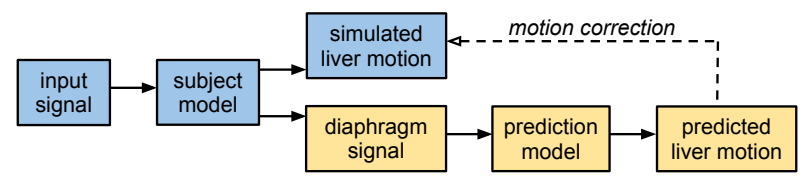

Fig. 2. Schematic of computations performed for the MR-model-based motion compensation scenario 

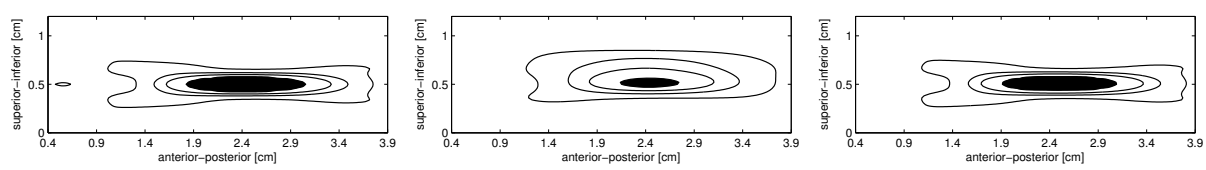

Fig. 3. Temperature contour plots of the temperature at $t^{\exp }=1.5 \mathrm{~s}$ of a single focus ablation with a heating rate of $r=40^{\circ} \mathrm{C} \mathrm{s}^{-1}$. The outer contour is at $40^{\circ} \mathrm{C}$, with a $5^{\circ} \mathrm{C}$ spacing between contours. The filled contour shows the extent of the lesion corresponding to a thermal dose TD $\geq 240$ minutes. Shown are the static case (left), gated (middle), and motion-compensated approach (right).

the focus was centred on the extent of the liver in the lateral direction. The locations were then set to $20 \%, 50 \%$, and $80 \%$ of the extent of the liver in the inferior-superior direction. For each subject, simulations of single focus HIFU heating were performed for four different combinations of heating rate $r=q / \rho C$ and exposure time $t^{\exp }$, for both the gated and motion-compensated approaches. In all simulations, a sinusoidal function with a breathing period of 4 seconds, representative of actual observed subject breathing, was used as an input signal for the subject model. The ablated volume ratio with respect to the static case, denoted by $\mathcal{V}^{\mathrm{ab}}$, was determined for all three ablation locations within the liver.

\section{Results}

By analysing the registration results at the anatomical landmark locations for all volunteers, we found a mean displacement of $7.4 \mathrm{~mm}$ with a standard deviation of $5.9 \mathrm{~mm}$, and a maximum displacement of $27.2 \mathrm{~mm}$. After registration, the mean TRE was $1.7 \mathrm{~mm}$, with a standard deviation of $1.2 \mathrm{~mm}$, and a maximum of $8.4 \mathrm{~mm}$. These results indicate that the registration accurately captures the respiratory motion from the sets of dynamic MR scans. As an example, Fig. 3 shows heating contour plots for a single focus ablation after 1.5 seconds of heating at a rate of $40^{\circ} \mathrm{Cs}^{-1}$. For this particular case, the gated approach is insufficient to produce a properly ablated region, as can be seen from the spread of the temperature profile and much smaller ablated volume. In contrast, the motion-compensated method almost fully recovers the ablated volume of the static case. Table 2 presents the motion amplitudes at the three chosen treatment locations for all subjects as computed from the normal breathing motion models, together with the residual amplitudes after this motion was corrected for using the deeper breathing guidance motion models. Table 3 summarises the results for the ablated volume ratios $\mathcal{V}^{\mathrm{ab}}$ relative to the equivalent static cases for the gated and motion-compensated scenarios for four combinations of heating rate and exposure time. For each volunteer, results are given for three different locations, distributed over the superior-inferior extent of the liver. To achieve an ablated volume ratio $\mathcal{V}^{\mathrm{ab}}>90 \%$ relative to the static case, required an exposure time $t^{\exp }<0.5 \mathrm{~s}$ and a heating rate $r>120^{\circ} \mathrm{C} / \mathrm{s}$ for the gated approach, whereas for the motion-compensated method $t^{\exp }<1 \mathrm{~s}$ and $r>60^{\circ} \mathrm{C} / \mathrm{s}$. 
Table 2. Maximum motion amplitudes in mm occurring during the simulations with the longest exposure times of $t^{\exp }=2 \mathrm{~s}$, for all subjects. Amplitudes of simulated respiratory motion as they would occur during gated treatments are given in the first row. The second row shows residual displacement amplitudes after a second motion model was used to compensate for the subject motion.

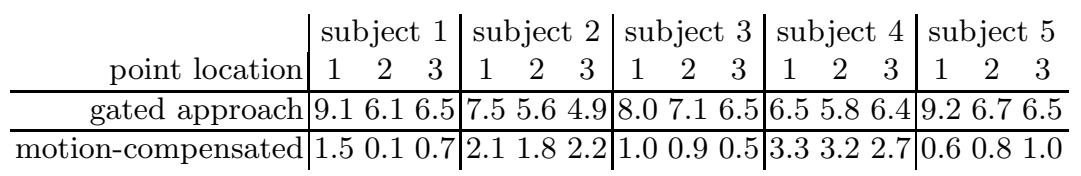

Table 3. Ablated volume ratios $\mathcal{V}^{\mathrm{ab}}$ expressed as a percentage relative to the equivalent static case for each subject, are given as a function of heating rates $r$ and total exposure times $t^{\exp }$ for three different locations within the liver. Results for conventional gated and MR-model-based motion compensation approaches are shown.

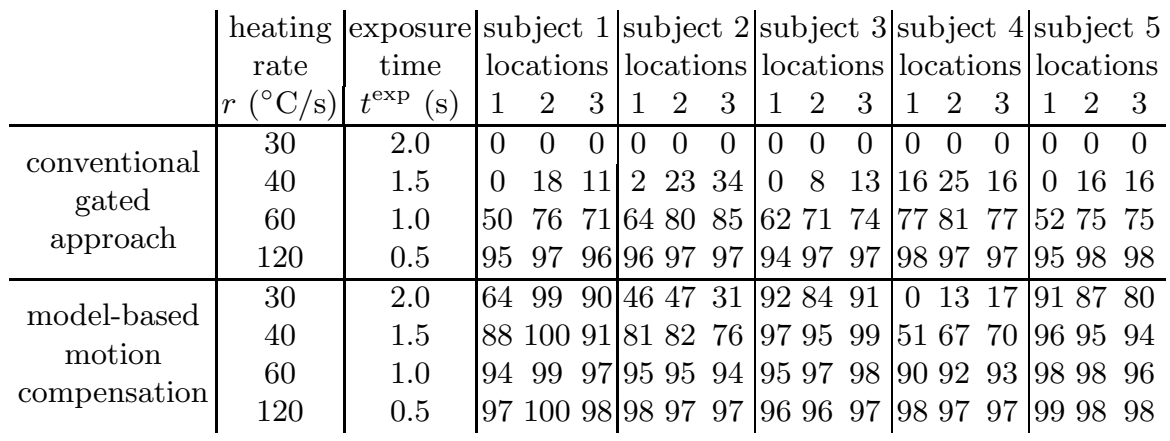

\section{Discussion and Conclusions}

We have presented detailed simulations of HIFU heating in the presence of realistic respiratory liver motion, for both gated and MR-model-based motioncompensated ablation. For the gated simulations, we found that the ablated volume increases when shorter HIFU exposure times, and correspondingly higher heating rates, were used. This is intuitive, since the target moves less when shortening the exposure time, and therefore less spread occurs in the applied heating pattern. Furthermore, from the results of Tables 2 and 3 , the largest liver motion occurred at the most superior location in the liver, resulting in consistently smaller ablated volumes than for more inferior located points in the liver. For the motion-compensated method, the ablated volume sizes are much less sensitive to variations in exposure time and heating rate, as long as $t^{\exp }<1 \mathrm{~s}$ and $r>60^{\circ} \mathrm{C} / \mathrm{s}$.

Although the use of a simple spherical bowl transducer is sufficient for the purpose of this initial study, we are planning to incorporate more realistic phased array pressure fields to simulate beam steering while compensating for the effects 
of ribs in the beam path. We conclude that model-based motion compensation allows for two times lower heating rates than gating, thereby reducing the risk of negative side-effects such as skin burns and focal boiling.

Acknowledgements. We would like to thank Tobias Schäffter for performing the MR scanning, and all volunteers for participating in this study. This work was supported by the EPSRC, grant numbers EP/F025750/1 and EP/F029217/1.

\section{References}

1. Pernot, M., Tanter, M., Fink, M.: 3-D real-time motion correction in high-intensity focused ultrasound therapy. Ultrasound in Medicine \& Biology 30(9), 1239 (2004)

2. de Oliveira, P.L., de Senneville, B.D., Dragonu, I., Moonen, C.T.W.: Rapid motion correction in MR-guided high-intensity focused ultrasound heating using real-time ultrasound echo information. NMR in Biomedicine 23(9), 1103-1108 (2010)

3. Ries, M., de Senneville, B.D.D., Roujol, S., Berber, Y., Quesson, B., Moonen, C.: Real-time 3D target tracking in MRI guided focused ultrasound ablations in moving tissues. Magnetic Resonance in Medicine (2010)

4. von Siebenthal, M., Székely, G., Gamper, U., Boesiger, P., Lomax, A., Cattin, P.: $4 \mathrm{D}$ MR imaging of respiratory organ motion and its variability. Physics in Medicine and Biology 52(6), 1547-1564 (2007)

5. Rijkhorst, E.-J., Heanes, D., Odille, F., Hawkes, D., Barratt, D.: Simulating dynamic ultrasound using MR-derived motion models to assess respiratory synchronisation for image-guided liver interventions. In: Navab, N., Jannin, P. (eds.) IPCAI 2010. LNCS, vol. 6135, pp. 113-123. Springer, Heidelberg (2010)

6. Crum, W.R., Tanner, C., Hawkes, D.J.: Anisotropic multi-scale fluid registration: evaluation in magnetic resonance breast imaging. Physics in Medicine and Biology 50(21), 5153-5174 (2005)

7. Pennes, H.H.: Analysis of tissue and arterial blood temperatures in the resting human forearm. Journal of Applied Physiology 1(2), 93-122 (1948)

8. Meaney, P.M., Clarke, R.L., ter Haar, G.R., Rivens, I.H.: A 3-D finite-element model for computation of temperature profiles and regions of thermal damage during focused ultrasound surgery exposures. Ultrasound in Medicine \& Biology 24(9), 1489-1499 (1998)

9. Sapareto, S.A., Dewey, W.C.: Thermal dose determination in cancer therapy. International Journal of Radiation Oncology*Biology*Physics 10(6), 787-800 (1984)

10. Williams, L.R., Leggett, R.W.: Reference values for resting blood flow to organs of man. Clin. Phys. Physiol. Meas. 10, 187 (1989) 